\title{
Optical properties of the Holstein-t- $J$ model from dynamical mean-field theory
}

\author{
E. Cappelluti a,b,*, S. Ciuchi ${ }^{\mathrm{c}}, \mathrm{S}^{\mathrm{a}}$ Fratini ${ }^{\mathrm{d}}$ \\ "Dipartimento di Fisica, Università "La Sapienza", P.le A. Moro 2, 00185 Rome, Italy \\ b $S M C$ Research Center and ISC, INFM-CNR, v. dei Taurini 19, 00185 Rome, Italy \\ ${ }^{\mathrm{c}}$ INFM and Dipartimento di Fisica, Università dell'Aquila, via Vetoio, I-67010 Coppito-L'Aquila, Italy \\ d Institut Néel - CNRS \& Université Joseph Fourier, BP 166, F-38042 Grenoble Cedex 9, France
}

\section{Abstract}

We employ dynamical mean-field theory to study the optical conductivity $\sigma(\omega)$ of one hole in the Holstein- $t$ - $J$ model. We provide an exact solution for $\sigma(\omega)$ in the limit of infinite connectivity. We apply our analysis to $\mathrm{Nd}_{2-x} \mathrm{Ce}_{x} \mathrm{CuO}_{4}$. We show that our model can explain many features of the optical conductivity in this compounds in terms of magnetic/lattice polaron formation.

Key words: magnetic/lattice polarons, spin fluctuations, optical conductivity, cuprates.

PACS: 71.10.Fd, 71.38.-k, 78.20.Bh, 75.30.Ds.

The problem of a single hole in the $t$ - $J$ model interacting also with the lattice degrees of freedom has attracted recently a notable interest in connection with the physical properties of the underdoped high- $T_{c}$ cuprates $[1,2,3,4]$. An important issue in this regime is the formation of lattice or magnetic polarons (or both of them) and their mutual interaction. Along this line, the one-particle properties (as the effective mass, spectral function, etc.) have been widely investigated with different techniques. Much less effort has been however paid to the study of the optical properties. On the analytical ground, the definition of the optical conductivity (OC) in the single hole is a delicate matter which needs particular care even for the pure $t-J$ or Holstein model $[5,6]$. On the other hand, numerical calculations on clusters are limited by finite size effects [7]. As a general rule, thus, the choice of a particular theoretical approach depends on which property is under examination and on its feasibility to investigate it.

In this paper we summarize the main results of our work based on the dynamical mean-field theory (DMFT). Technical details will be presented in a forthcoming longer publication [8]. In the infinite coordination number limit $z \rightarrow$ $\infty$, we provide an exact solution for $\sigma(\omega)$ as a functional of the local one-particle Green's function at finite temperature. It should be stressed that, due to the classical treatment of the magnetic background, the DMFT solution for

\footnotetext{
* Corresponding author. Tel: (+39) 06-49937453 fax: (+39) 0649937440

Email address: emmcapp@roma1.infn.it (E. Cappelluti).
}

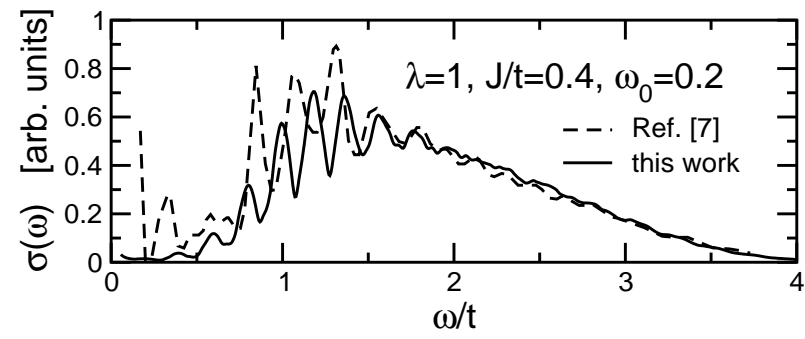

Fig. 1. Comparison between the optical conductivity $\sigma(\omega)$ obtained by our DMFT solution and Lanczos diagonalization in two dimensions on a finite cluster (Ref. [7]).

$z \rightarrow \infty$ is purely local so that it cannot describe the coherent propagation of holes due to the spin fluctuations, nor the metallic Drude-like peak in $\sigma(\omega)$. On the other hand, the local properties (as the average number of phonons, size of the magnetic polaron, etc.) are well captured by this approach, [9] as well as the incoherent contributions to the OC. We can explicitly show this feature by comparing in Fig. 1 our DMFT results with numerical calculations using Lanczos diagonalization for a single hole in the 2D Holstein$t$ - $J$ model on a $\sqrt{10} \times \sqrt{10}$ cluster [7].

The remarkably good agreement of the overall shape assesses the feasibility of our approach to investigate the incoherent contributions to the finite frequency OC. This issue is particularly important in light of the intensive debate about the origin of the mid-infrared (MIR) band in the underdoped high- $T_{c}$ cuprates. Different interpretations for this feature have been discussed in the literature, involving 
charge/spin fluctuations, stripe ordering, and other mechanisms. This spread of different mechanisms reflects the presence in this doping regime of several actors, which makes it difficult to isolate each effect from the others. A simpler and ideal situation is the case of electron-doped cuprates, as $\mathrm{Nd}_{2-x} \mathrm{Ce}_{x} \mathrm{CuO}_{4}$. In these compounds, the long-range antiferromagnetic $(\mathrm{AF})$ order extents up to $x \simeq 0.14$, so that the low doping regime $x \lesssim 0.1$ we are interested in, lies well within the AF phase. On the experimental side, in addition, a detailed and exhaustive study of the optical conductivity as a function of temperature $T$ and of the doping $x$ was recently provided in Ref. [10]. In that work the authors showed that the low doping OC spectra are characterized at low temperature by a MIR pseudogap, with an absorption band edge which varies from $E_{\mathrm{MIR}} \simeq 0.5-0.6$ for $x=$ 0.05 to $E_{\mathrm{MIR}} \simeq 0.3-0.4$ for $x=0.1$, and is barely distinguishable for $x=0.125$. Quite interestingly, increasing the temperature leads to a filling of the pseudogap, rather than a closing of it. Also remarkable is the temperature dependence of the MIR spectral weight which does not present any signature at the long-range Néel temperature $T_{\mathrm{N}}$ but rather a kink to a higher "pseudogap" temperature $T^{*}$.

We show here that our approach is able to describe all these features, and in particular the MIR band edge, in terms of an optical gap due to the formation of a magnetic/lattice polaron. We define $T^{*}$ as the temperature where the size of the spin polaron becomes larger than the AF correlation length, that is the maximum temperature where an injected charge actually probes the magnetic background. In this perspective we can identify $T^{*}$ with the mean field Néel temperature of our model, which represents the temperature above which the system is described by a paramagnetic state (rather than the onset of long range order). From Ref. [10] we get for instance $T^{*}=440 \mathrm{~K}$ at $x=0.05$ and $T^{*}=200 \mathrm{~K}$ at $x=0.125$. Using the CurieWeiss relation $T_{\mathrm{MF}}^{*}=J / 4$ we estimate respectively $J=152$ $\mathrm{meV}(J / t=0.126)$ and $J=69 \mathrm{meV}(J / t=0.057)$. Note that such values of $J$ do not represent the bare exchange interaction but rather the effective spin-exchange coupling which is reduced by hole doping. We also set $\omega_{0}=84 \mathrm{meV}$, consistent with the energy window of the optical phonons in the cuprates. The electron-phonon (el-ph) coupling constant is fixed to $\lambda=0.75$ in order to reproduce the experimental MIR band edge $\approx 0.5-0.6 \mathrm{eV}$ in the optical conductivity at $x=0.05$, and we assume $\lambda$ to be independent of the doping $x$. Note that with these choices no more free adjustable parameters remain.

In Fig. 2 we show the temperature evolution of the MIR optical conductivity for the representative cases $x=0.05$ and $x=0.125$ (note that in order to compare with the experimental data of Ref. [10] the tail of a Drude-peak should be superimposed). Most remarkable is the behavior of $\sigma(\omega)$ at low temperature, which shows a well defined gap for $x=0.05$ while no gap is found for $x=0.125$. This feature reflects the formation of the lattice polaron and its interplay with the spin degrees of freedom. While the el-ph coupling $\lambda=0.75$ alone is not strong enough at $x=0.125$

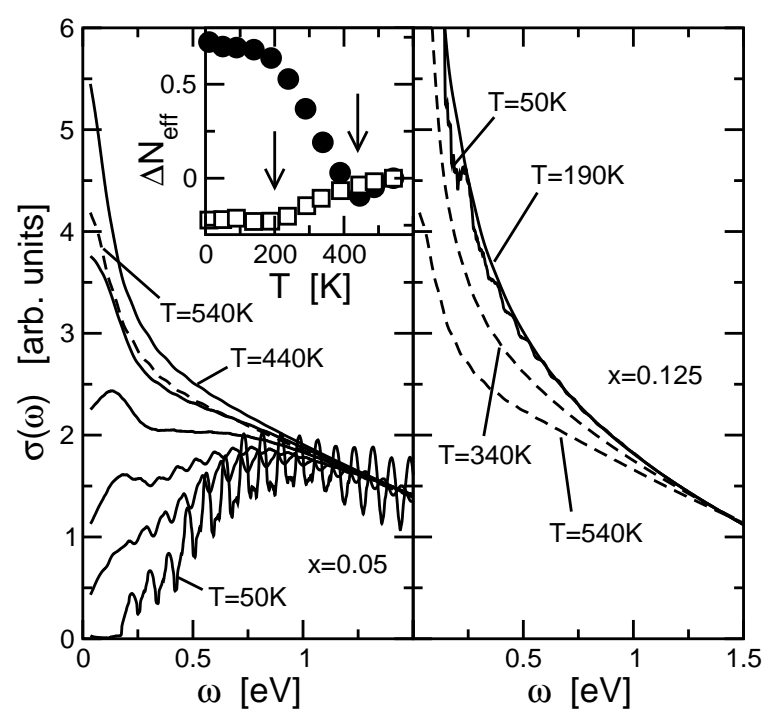

Fig. 2. Temperature dependence of the optical conductivity $\sigma(\omega)$ for $x=0.05$ and $x=0.125$. Solid lines are used for $T \leq T^{*}$, dashed lines for $T>T^{*}$. Inset: loss of the MIR spectral weight $\Delta N_{\text {eff }}$, as defined in Ref. [10], as function of $T$ for $x=0.05$ (filled circles) and $x=0.125$ (empty squares). Arrows mark the corresponding $T^{*}$.

$(J / t=0.057)$ to establish a spin/lattice polaron, the localization effects induced by the larger exchange coupling $J / t=0.126$ at $x=0.05$ favor the lattice polaron formation. This leads thus to the opening of an optical gap in $\sigma(\omega)$ (this key point will be extensively discussed in a forthcoming publication[8]). Increasing $T$ reduces the localization effects induced by the magnetic ordering. This makes the positive interplay with the el-ph coupling less effective, leading to a progressive filling of the pseudogap. Note that this effect disappears in the disordered magnetic case for $T>T^{*}$, and further increasing of $T$ leads to a reduction of the MIR optical conductivity which is spread on a larger energy window. This is reflected in the characteristic temperature behavior of the MIR spectral weight $\Delta N_{\text {eff }}$, as defined in Ref. [10], which presents a kink at $T^{*}$ (inset of Fig. $2)[11]$.

\section{References}

[1] A.S. Mishchenko and and N. Nagaosa, Phys. Rev. Lett. 93 (2004) 0236402; Phys. Rev. B 73 (2006) 092502.

[2] O. Rösch and O. Gunnarsson, Phys. Rev. Lett. 92 (2004) 146403 Eur. Phys. J. B 43 (2005) 11.

[3] O. Gunnarsson and O. Rösch, Phys. Rev. B 73 (2006) 174521.

[4] P. Prelovšek, R. Zeyher, and P. Horsch, Phys. Rev. Lett. 96 (2006) 086402.

[5] M.P.H. Stumpf and D.E. Logan, Eur.Phys.J.B, 8 (1999) 377.

[6] S. Fratini and S. Ciuchi, Phys. Rev. B 74 (2006) 075101.

[7] B. Bäuml et al., Phys. Rev. B 58 (1998) 3663.

[8] E. Cappelluti, S. Ciuchi and S. Fratini, in preparation (2007).

[9] E. Cappelluti and S. Ciuchi, Phys. Rev. B 66 (2002) 165102.

[10] Y. Onose et., Phys. Rev. B 69 (2004) 024504.

[11] Since we do not find any isosbestic point in our calculations, we use the experimental energy windows of Ref. [10] to define 
$\Delta N_{\text {eff }}$, namely $\omega_{\min }=0.12 \mathrm{eV}, \omega_{\max }=0.42 \mathrm{eV}$ for $x=0.05$ and $\omega_{\max }=0.21 \mathrm{eV}$ for $x=0.125$. 